\title{
Delayed projection neural network for solving degenerate quadratic program
}

\author{
YANG Yong \\ Chong Qing Creation Vocational Colleg \\ Chong Qing yongchuan \\ yy13594196329@163.com
}

\author{
ZHANG Jing-wen \\ Chong Qing Creation Vocational Colleg \\ Chong Qing yongchuan \\ wenwen80521@126.com
}

\begin{abstract}
A delayed projection neural network model is presented for solving degenerate quadratic programming problem with general linear constraints. By constructing suitable Lyapunov functions, the proposed neural network is proved to be global convergent to the equilibrium points set. Compared with the existing neural networks for solving strict convex quadratic program, the proposed delayed neural network for solving degenerate convex ${ }^{1}$ quadratic program has wider domain for implementation. The transient behavior of the neural network is simulated and the validity of the result obtained in this paper is verified with an illustrative example.
\end{abstract}

Keywords-delayed neural networks; projection equation; quadratic optimization;global exponential stability; optimal solution (key words)

\section{INTRODUCTION}

It is well known that the quadratic optimization problems

$$
\frac{1}{2} x^{\mathrm{T}} Q x+a^{\mathrm{T}} x, \quad x \in \Omega,
$$

where $Q \in R^{n \times n}, a \in R^{n}, \Omega$ is a convex set, arise in a wide variety of scientific and engineering applications including regression analysis, image and signal progressing, parameter estimation, filter design and robust control[1]. In many real time applications th. The main advantage of neural network.

\section{A DELAYED PROJECTION NEURAL NETWORK}

In this paper, we present a new delayed projection neural network for solving the following degenerate convex quadratic programming problem

$$
\min \frac{1}{2} x^{\mathrm{T}} M x+a^{\mathrm{T}} x
$$$$
\text { subjectto } d \leq D x \leq h \text {, }
$$

where $d, h \in R^{n}, \boldsymbol{D}$ is an $n \times n$ matrix. the authors proposed a delayed projection neural network for solving the following degenerate quadratic problem[3].

$$
\min \frac{1}{2} x^{\mathrm{T}} M x+a^{\mathrm{T}} x
$$

subject to $A x=b, x \geq 0$

where $\boldsymbol{M}$ is a positive semi-definite matrix and $\boldsymbol{A}$ is a full row rank matrix. Their neural network was proved to be global exponentially stable and could converge to an optimal solution of their optimization problem.

Proposition 1 Let $u^{*}$ be a solution of the project equation

$$
\left.u=P_{\Omega}\left(u-\left(D^{\mathrm{T}}\right)^{-1} M D^{-1} u-\left(D^{\mathrm{T}}\right)^{-1} a\right)\right), \text { then, } x^{*} \text { is an }
$$
optimal solution of (2).

Proposition $2 x^{*}$ is a solution to problem (2) if and only if $u^{*}$ is a zero point of the following map:

$$
\left.G(u)=P_{\Omega}\left(u-\left(D^{\mathrm{T}}\right)^{-1} M D^{-1} u-\left(D^{\mathrm{T}}\right)^{-1} a\right)\right)-u
$$

In the following, we propose a neural network ${ }^{[2]-[4]}$, which is said to be the delayed projection neural network for solving (2) and (3),

$$
\begin{aligned}
\frac{d x(t)}{d t}= & -2 x(t)+P_{\Omega}\left(x(t)-\left(\left(D^{\mathrm{T}}\right)^{-1} M D^{-1} x(t)\right.\right. \\
& \left.\left.+\left(D^{\mathrm{T}}\right)^{-1} a\right)\right)+x(t-\tau)
\end{aligned}
$$

where $\tau \geq 0$ denotes the transmission delay, $\mathrm{P}_{\Omega}$ is a projection operator

\section{CONVERGENCE RESULTS}

In this section, we will give the convergence results of the proposed neural network (4). To do so, we need to introduce the following lemmas and proposition.

Lemma 1[4]If $\Omega \subset R^{n}$ is a closed convex subset and $z \in R^{n}$ is a given point, then there exists $x \in \Omega$ such that $(x-z, y-x) \geq 0$, if and only if $x=p_{\Omega}(z)$, where $P_{\Omega}$ is a project function on $\Omega$, and given by $P_{\Omega}(u)=\arg \min _{y \in \Omega}\|u-y\|$.

Lemma2Let $A \in R^{n \times n}$ and $B \in R^{n \times n}$, then $\lambda$ is a nonzero eigenvalue of $A B$ if and only if it is a nonzero eigenvalue of $B A$. 
Lemma3 For each $\varphi(t) \in C \stackrel{\Delta}{=} C([-\tau, 0])$, the whole of time interval $[0, \infty),(2)$ there exists a unique continuous solution.

Proposition2.1 Let $I_{n}$ is unit matrix, $M$ is a positive semi-definite matrix and symmetric. Then $I_{n}+\left(D^{\mathrm{T}}\right)^{-1} M D^{-1}$ is a positive definite matrix and symmetric.

Proof In order to prove that the matrix

$$
I_{n}+\left(D^{\mathrm{T}}\right)^{-1} M D^{-1}
$$

is a positive definite matrix, we only need to prove that the matrix

$$
\left(D^{\mathrm{T}}\right)^{-1} M D^{-1}
$$

is a positive semi-definite matrix and symmetric. Obviously, it is a symmetric.

We denote $\lambda\left[\left(D^{\mathrm{T}}\right)^{-1} M D^{-1}\right]$ as the set consisted of the nonzero eigenvalues of $\left(D^{\mathrm{T}}\right)^{-1} M D^{-1}$. Then according to Lemma3, we get that

$$
\lambda\left[\left(D^{\mathrm{T}}\right)^{-1} M D^{-1}\right]=\lambda\left[D^{-1}\left(D^{\mathrm{T}}\right)^{-1} M\right]
$$

Because $D^{-1}\left(D^{\mathrm{T}}\right)^{-1} M$ is a positive semi-definite matrix, $\left(D^{\mathrm{T}}\right)^{-1} M D^{-1}$ is a positive semi-definite matrix, too. Then is $I_{n}+\left(D^{\mathrm{T}}\right)^{-1} M D^{-1}$ a positive definite matrix and symmetric.

Theorem2.2Delayed neural network (4) is global convergence to the solutions set of problem(2), when the matrix $\mathrm{M}$ is a positive semi- definite matrix and symmetric.

Proof Assume $x^{*}$ is an equilibrium point of system(4). Consider the following Lyapunov-Krasovskii functional

$$
\begin{aligned}
V(x(t))= & V_{0}(x(t))+\frac{1}{2} \int_{t-\tau}^{t}\left(x(s)-x^{*}\right)^{\mathrm{T}} \\
& \left(\left(D^{\mathrm{T}}\right)^{-1} M D^{-1}+I\right)\left(x(s)-x^{*}\right) d s
\end{aligned}
$$

where I is n-dimensional identity matrix,

$$
\begin{gathered}
V_{0}(x(t))=f(x)-f\left(x^{*}\right)-<f^{\prime}\left(x^{*}\right), x-x^{*}> \\
+\frac{1}{2}\left\|x-x^{*}\right\|^{2}, \\
\left.f(x)=\frac{1}{2} x^{\mathrm{T}} D^{\mathrm{T}}\right)^{-1} M D^{-1} x+a^{\mathrm{T}} x, \\
\text { then } f^{\prime}(x)=D M x+a . \\
\frac{d V_{0}(x(t))}{d t}=<f^{\prime}(x)-f^{\prime}\left(x^{*}\right), \frac{d x}{d t}>-<x-x^{*}, \frac{d x}{d t}> \\
=<f^{\prime}(x)-f^{\prime}\left(x^{*}\right), \\
P_{X}\left[x(t)-\left(\left(D^{\mathrm{T}}\right)^{-1} M D^{-1} x(t)+\left(D^{\mathrm{T}}\right)^{-1} a\right)\right]-x(t)> \\
+<f^{\prime}(x)-f^{\prime}\left(x^{*}\right),-x(t)+x(t-\tau)>
\end{gathered}
$$

$$
\begin{aligned}
& +<x-x^{*}, \\
& \begin{array}{l}
P_{X}\left[x(t)-\left(\left(D^{\mathrm{T}}\right)^{-1} M D^{-1} x(t)+\left(D^{\mathrm{T}}\right)^{-1} a\right)\right]-x(t)> \\
\quad+<x-x^{*},-x(t)+x(t-\tau)>
\end{array} \\
& \quad \text { the }<f^{\prime}(x)-f^{\prime}\left(x^{*}\right), \\
& P_{X}\left[x(t)-\left(\left(D^{\mathrm{T}}\right)^{-1} M D^{-1} x(t)+\left(D^{\mathrm{T}}\right)^{-1} a\right)\right]-x(t)> \\
& =<f^{\prime}(x)-f^{\prime}\left(x^{*}\right), \\
& P_{X}\left[x(t)-\left(\left(D^{\mathrm{T}}\right)^{-1} M D^{-1} x(t)+\left(D^{\mathrm{T}}\right)^{-1} a\right)\right]-x^{*}> \\
& +<f^{\prime}(x)-f^{\prime}\left(x^{*}\right), x^{*}-x> \\
& =<f^{\prime}(x), \\
& P_{X}\left[x(t)-\left(\left(D^{\mathrm{T}}\right)^{-1} M D^{-1} x(t)+\left(D^{\mathrm{T}}\right)^{-1} a\right)\right]-x^{*}> \\
& -<f^{*}(x), P_{X}\left[x(t)-\left(\left(D^{\mathrm{T}}\right)^{-1} M D^{-1} x(t)+\left(D^{\mathrm{T}}\right)^{-1} a\right)\right] \\
& -x^{*}>+<f^{\prime}(x)-f^{\prime}\left(x^{*}\right), x^{*}-x>
\end{aligned}
$$

It can follows that

$<f^{\prime}(x)$,

$\left.P_{X}[x t)-\left(\left(D^{\mathrm{T}}\right)^{-1} M D^{-1} x(t)+\left(D^{\mathrm{T}}\right)^{-1} a\right)\right]-x^{*}>$

$=<f^{\prime}(x)-x(t)+$

$P_{X}\left[x(t)-\left(\left(D^{\mathrm{T}}\right)^{-1} M D^{-1} x(t)+\left(D^{\mathrm{T}}\right)^{-1} a\right)\right]$

$P_{X}\left[x(t)-\left(\left(D^{\mathrm{T}}\right)^{-1} M D^{-1} x(t)+\left(D^{\mathrm{T}}\right)^{-1} a\right)\right]-x^{*}>$

$+<x(t)-P_{X}\left[x(t)-\left(\left(D^{\mathrm{T}}\right)^{-1} M D^{-1} x(t)+\left(D^{\mathrm{T}}\right)^{-1} a\right)\right]$

$P_{X}\left[x(t)-\left(\left(D^{\mathrm{T}}\right)^{-1} M D^{-1} x(t)+\left(D^{\mathrm{T}}\right)^{-1} a\right)\right]-x^{*}>$

$=<f^{\prime}(x)-x(t)+$

$P_{X}\left[x(t)-\left(\left(D^{\mathrm{T}}\right)^{-1} M D^{-1} x(t)+\left(D^{\mathrm{T}}\right)^{-1} a\right)\right]$

$P_{X}\left[x(t)-\left(\left(D^{\mathrm{T}}\right)^{-1} M D^{-1} x(t)+\left(D^{\mathrm{T}}\right)^{-1} a\right)\right]-x^{*}>$

$+<x(t)-P_{X}\left[x(t)-\left(\left(D^{\mathrm{T}}\right)^{-1} M D^{-1} x(t)+\left(D^{\mathrm{T}}\right)^{-1} a\right)\right]$,

$P_{X}\left[x(t)-\left(\left(D^{\mathrm{T}}\right)^{-1} M D^{-1} x(t)+\left(D^{\mathrm{T}}\right)^{-1} a\right)\right]-x(t)>$

$+<x(t)-P_{X}\left[x(t)-\left(\left(D^{\mathrm{T}}\right)^{-1} M D^{-1} x(t)+\left(D^{\mathrm{T}}\right)^{-1} a\right)\right]$

$x(t)-x^{*}>$

Combining (5)-(6)

$$
\begin{aligned}
& \frac{d V_{0}(x(t))}{d t} \\
= & <f^{\prime}(x)-x(t)+P_{X}[x(t)-(M x(t)+a)], \\
& P_{X}[x(t)-(M x(t)+a)]-x^{*}> \\
- & \left\|P_{X}\left[x(t)-\left(\left(D^{\mathrm{T}}\right)^{-1} M D^{-1} x(t)+\left(D^{\mathrm{T}}\right)^{-1} a\right)\right]\right\|^{2}
\end{aligned}
$$




$$
\begin{aligned}
& +<x(t)-P_{X}\left[x(t)-\left(\left(D^{\mathrm{T}}\right)^{-1} M D^{-1} x(t)+\left(D^{\mathrm{T}}\right)^{-1} a\right)\right] \\
& x(t)-x^{*}> \\
& +<f^{\prime}(x)-f^{\prime}\left(x^{*}\right),-x(t)+x(t-\tau)> \\
& -<f^{*}(x), P_{X}\left[x(t)-\left(\left(D^{\mathrm{T}}\right)^{-1} M D^{-1} x(t)+\left(D^{\mathrm{T}}\right)^{-1} a\right)\right]-x^{*}> \\
& +<f^{\prime}(x)-f^{\prime}\left(x^{*}\right), x^{*}-x> \\
& +<x(t)-x^{*}, \\
& P_{X}\left[x(t)-\left(\left(D^{\mathrm{T}}\right)^{-1} M D^{-1} x(t)+\left(D^{\mathrm{T}}\right)^{-1} a\right)\right]-x(t)> \\
& +<x-x^{*},-x(t)+x(t-\tau> \\
& \leq-\left\|x(t)-P_{X}\left[x(t)-\left(\left(D^{\mathrm{T}}\right)^{-1} M D^{-1} x(t)+\left(D^{\mathrm{T}}\right)^{-1} a\right)\right]\right\|^{2} \\
& +<x-x^{*},-x(t)+x(t-\tau> \\
& +<f^{\prime}(x)-f^{\prime}\left(x^{*}\right),-x(t)+x(t-\tau)> \\
& =-\left\|x(t)-P_{X}\left[x(t)-\left(\left(D^{\mathrm{T}}\right)^{-1} M D^{-1} x(t)+\left(D^{\mathrm{T}}\right)^{-1} a\right)\right]\right\|^{2} \\
& +<\left(D^{\mathrm{T}}\right)^{-1} M D^{-1}\left(x-x^{*}\right),-x(t)+x(t-\tau)> \\
& +<x-x^{*},-x(t)+x(t-\tau> \\
& = \\
& +\left\|x(t)-P_{X}\left[x(t)-\left(\left(D^{\mathrm{T}}\right)^{-1} M D^{-1} x(t)+\left(D^{\mathrm{T}}\right)^{-1} a\right)\right]\right\|^{2} \\
& +<\left(\left(D^{\mathrm{T}}\right)^{-1} M D^{-1}+I\right)\left(x(t)-x^{*}\right),-x(t)+x(t-\tau>
\end{aligned}
$$

Therefore

$$
\begin{aligned}
& \frac{d V\left(x_{t}\right)}{d t}=\frac{d V_{0}(x(t))}{d t} \\
& +\frac{1}{2}\left(x(t)-x^{*}\right)^{\mathrm{T}}\left(\left(D^{\mathrm{T}}\right)^{-1} M D^{-1}+I\right)\left(x(t)-x^{*}\right) \\
& -\frac{1}{2}\left(x(t-\tau)-x^{*}\right)^{\mathrm{T}}\left(\left(D^{\mathrm{T}}\right)^{-1} M D^{-1}+I\right)\left(x(t-\tau)-x^{*}\right) \\
& \leq-\left\|x(t)-P_{X}\left[x(t)-\left(\left(D^{\mathrm{T}}\right)^{-1} M D^{-1} x(t)+\left(D^{\mathrm{T}}\right)^{-1} a\right)\right]\right\|^{2} \\
& +<\left(\left(D^{\mathrm{T}}\right)^{-1} M D^{-1}+I\right)\left(x(t)-x^{*}\right),-x(t)+x(t-\tau> \\
& +\frac{1}{2}\left(x(t)-x^{*}\right)^{\mathrm{T}}\left(\left(D^{\mathrm{T}}\right)^{-1} M D^{-1}+I\right)\left(x(t)-x^{*}\right) \\
& -\frac{1}{2}\left(x(t-\tau)-x^{*}\right)^{T}\left(\left(D^{T}\right)^{-1} M D^{-1}+I\right)\left(x(t-\tau)-x^{*}\right) \\
& =-\left\|x(t)-P_{X}\left[x(t)-\left(\left(D^{\mathrm{T}}\right)^{-1} M D^{-1} x(t)+\left(D^{\mathrm{T}}\right)^{-1} a\right)\right]\right\|^{2} \\
& +<\left(\left(D^{\mathrm{T}}\right)^{-1} M D^{-1}+I\right)\left(x(t)-x^{*}\right),
\end{aligned}
$$

$$
\begin{aligned}
& +\frac{1}{2}\left(x(t)-x^{*}\right)^{\mathrm{T}}\left(\left(D^{\mathrm{T}}\right)^{-1} M D^{-1}+I\right)\left(x(t)-x^{*}\right) \\
& -\frac{1}{2}\left(x(t-\tau)-x^{*}\right)^{\mathrm{T}}\left(\left(D^{\mathrm{T}}\right)^{-1} M D^{-1}+I\right)\left(x(t-\tau)-x^{*}\right) \\
& =-\left\|x(t)-P_{X}\left[x(t)-\left(\left(D^{\mathrm{T}}\right)^{-1} M D^{-1} x(t)+\left(D^{\mathrm{T}}\right)^{-1} a\right)\right]\right\|^{2} \\
& -\frac{1}{2}\left(x(t)-x^{*}\right)^{\mathrm{T}}\left(\left(D^{\mathrm{T}}\right)^{-1} M D^{-1}+I\right)\left(x(t)-x^{*}\right) \\
& +\left(x(t)-x^{*}\right)^{\mathrm{T}}\left(\left(D^{\mathrm{T}}\right)^{-1} M D^{-1}+I\right)\left(x(t-\tau)-x^{*}\right) \\
& -\frac{1}{2}\left(x(t-\tau)-x^{*}\right)^{\mathrm{T}}\left(\left(D^{\mathrm{T}}\right)^{-1} M D^{-1}+I\right)\left(x(t-\tau)-x^{*}\right)
\end{aligned}
$$

By Proposition3.1, $\left(D^{\mathrm{T}}\right)^{-1} M D^{-1}+I$ is a symmetric and positive definite matrix, So there exists a symmetric matrix $\mathrm{Q}$, Such that $D M+I=Q^{2}$.It follows that:

$$
\begin{aligned}
& \frac{d V\left(x_{t}\right)}{d t} \leq \\
&-\left\|x(t)-P_{X}\left[x(t)-\left(\left(D^{\mathrm{T}}\right)^{-1} M D^{-1} x(t)+\left(D^{\mathrm{T}}\right)^{-1} a\right)\right]\right\|^{2} \\
&-\left.\frac{1}{2}\left(Q x(t)-x^{*}\right)-Q\left(x(t-\tau)-x^{*}\right)\right)^{\mathrm{T}} \times\left(Q x(t)-x^{*}\right) \\
&-\left.Q\left(x(t-\tau)-x^{*}\right)\right) \\
&=-\left\|x(t)-P_{X}\left[x(t)-\left(\left(D^{\mathrm{T}}\right)^{-1} M D^{-1} x(t)+\left(D^{\mathrm{T}}\right)^{-1} a\right)\right]\right\|^{2} \\
&- \frac{1}{2}\left(Q(x(t)-Q(x(t-\tau)))^{\mathrm{T}} \times(Q x(t)-Q(x(t-\tau))\right. \\
&=-\left\|x(t)-P_{X}\left[x(t)-\left(\left(D^{\mathrm{T}}\right)^{-1} M D^{-1} x(t)+\left(D^{\mathrm{T}}\right)^{-1} a\right)\right]\right\|^{2} \\
&- \frac{1}{2}\left((x(t)-(x(t-\tau)))^{\mathrm{T}}\left(\left(D^{\mathrm{T}}\right)^{-1} M D^{-1}+I\right)(x(t)-(x(t-\tau))\right. \\
& \leq 0
\end{aligned}
$$

Therefore, $V\left(x_{t}\right)$ is a Lyapunov functional of system (4).From lemma 4,we have that the maximal existence interval of the solution of (4) is $[0, \infty)$. By the LaSalle invariance principle[5], there exists a constant $\tau$, such that $x_{t} \rightarrow E \cap V^{-1}(\tau), t \rightarrow \infty$, where $\mathrm{E}$ is the maximal invariant set in $S=\left\{x_{t}|d v / d t=0|\right\}$. Form (8) and the definition of $V\left(x_{t}\right)$, we have that $d v / d t=0$ if and only if $d v / d t=0$.It follows that: 


$$
E \cap\{x \in \Omega|d x / d t=0|\}
$$

So the dynamic system(4) is globally convergent to the equilibrium points set, which corresponds to the solutions of problem(2).This completes the proof.

\section{CONCLUSIONS}

we have developed a new class of delayed neural network for solving degenerate quadratic program, the equilibrium point of the proposed neural network is equivalent to the solution of quadratic programming problem. We have investigated the existence, global convergent of the equilibrium points for the delayed neural network. The results obtained in this paper are valuable in solving degenerate quadratic programming problem in engineering.

\section{REFERENCES}

[1] BAZARAA M S, Herali H D S, SHETTY C M. Nonlinear programming theory and algorithms [M].2nd ed. New York :Wiley, 1993.

[2] FENG G, WANG J.A recurrent neural network with exponential convergence for solving convex quadratic program and related linearpiecewise equation[J]. Neural Networks , 2004,17 :1003-1015.

[3] XIA Y S, Wang J. A general projection neural network for solving monotone variational inequalities and related optimizationproblems[J].

IEEE Trans Neural Networks,2004,15:318-328.

[4] BAIOCCHI C, CAPELO A. 1984. Variational and quasivariational inequalities

applications to free boundary problems[M]. NewYork:Wiley,1984.

[5] LASALLE J P.The stability of dynamical systems[M]. Philadelphia:SIMA, 1976. 